\title{
Low frequency sawtooth precursor activity in ASDEX Upgrade
}

\author{
G. Papp ${ }^{1,2}$, G. I. Pokol ${ }^{1}$, G. Pór ${ }^{1}$, A. Magyarkuti ${ }^{1}$, N. Lazányi ${ }^{1}$, \\ L. Horváth ${ }^{1}$, V. Igochine ${ }^{3}$, M. Maraschek ${ }^{3}$ and ASDEX Upgrade \\ Team $^{3}$ \\ ${ }^{1}$ Department of Nuclear Techniques, Budapest University of Technology and \\ Economics, Association EURATOM, Pf 91, H-1521 Budapest, Hungary \\ ${ }^{2}$ Department of Applied Physics, Nuclear Engineering, Chalmers University of \\ Technology and Euratom-VR Association, SE-41296 Göteborg, Sweden \\ ${ }^{3}$ Max-Planck-Institut für Plasmaphysik, Association EURATOM, D-85748 Garching, \\ Germany \\ E-mail: papp@reak.bme.hu
}

\begin{abstract}
Present paper describes the precursor activity observed in the ASDEX Upgrade tokamak before sawtooth crashes in various neutral beam heated plasmas, utilizing the soft X-ray diagnostic. Besides the well-known $(m, n)=(1,1)$ internal kink mode and its harmonics, a lower frequency mode is studied in detail. Power modulation of this mode is found to correlate with the power modulation of the $(1,1)$ kink mode in the quasi-stationary intervals indicating possible non-linear interaction. Throughout the studied sawtooth crashes, the power of the lower frequency mode rose by several orders of magnitude just before the crash. Besides its temporal behaviour, its spatial structure was estimated and the most likely value was found to be $(1,1)$. A possible role of this mode in the mechanism of the sawtooth crash is discussed.
\end{abstract}

PACS numbers: 52.35.-g, 52.35.Mw, 52.35.Py, 52.55.Fa, 52.70.-m, 52.70.La

Published as "G. Papp et al, Plasma Phys. Control. Fusion 53065007 (2011)"

http://stacks.iop.org/PPCF/53/065007 


\section{Introduction}

The sawtooth oscillation is a periodic collapse phenomenon widely observed in tokamaks. It develops in the plasma core when the safety factor $(q)$ on-axis is below 1 . In the core, the temperature and density ramp up slowly over most of the sawtooth period while they rapidly crash down in the remainder. During the crash phase there is an intensive density- and heat transport outwards. The temperature and density outside the core ramp up quickly just after the crash, and decrease gradually to its equilibrium value. This effect is most noticeable on the line integrated Soft X-Ray (SXR) measurements of the plasma core, where the repeating sequence of slow growth and sudden drop of intensity gives the characteristic sawtooth shape [1].

The sawtooth phenomenon is important for different reasons. It is predicted that fusion-born $\alpha$ particles will lead to long sawtooth periods on ITER [2,3]. The plasma can easily survive the small performance-reducing drops of the main core plasma parameters caused by sawteeth. It is the coupling of sawteeth to other, more harmful modes which is of most concern. Sawteeth has been shown to trigger Neoclassical Tearing Modes (NTMs) $[4,5]$, resulting in a substantial confinement degradation. In extreme cases, this may lead to a rapid termination of the plasma discharge (disruption). Disruptions and their consequences pose significant design and plasma operation challenges for reactorregime tokamaks [6]. Sawteeth have also been identified as possible threat to plasma self-heating [6]. As well as the above detrimental effects on the plasma performance, the sawtooth instability will remove helium ash and impurities from the core of burning plasmas, thereby preventing a degradation of the core temperature [7]. Thus the goal is to control the sawteeth, not to totally avoid them. For these reasons, significant effort has been placed by the community in observing, controlling and understanding the sawtooth instability.

Although the physical processes that govern sawtooth oscillations in fusion plasmas remain only partially understood, the knowledge of how to control sawteeth has improved significantly in recent years. Several methods have been shown to be suitable for sawtooth control $[8,9]$. Sawtooth control can be realized with a variety of heating and current drive techniques $[10,11,12,13,14,15]$. There are also promising results with real-time control systems [16]. When the plasma is heated using ICRH or NBI, the fast ions born in the plasma can significantly affect the stability of the $(1,1)$ internal kink mode [17]. The $(1,1)$ kink mode is a well known sawtooth precursor [18], which is characterized by $(m, n)=(1,1)$ spatial structure, where $m$ and $n$ are the poloidal and toroidal mode numbers, respectively. Most of the control methods rely on influencing the $(1,1)$ internal kink mode.

Despite the various results, the details of the crash mechanism itself still need to be revealed. Ever since the first, the Kadomtsev model [19] there have always been models which later on have been contradicted with experimental observations. The vast majority of the models take only the $(1,1)$ mode into account. Experimental results have 
showed that other modes are important at the crash phase. The importance of higher order harmonics, namely the $(2,2)$ and $(3,3)$ has been investigated on ASDEX Upgrade [20, 21, 22] and HT-7 [23]. These results fit well into the stochastic model [24, 25] of the sawtooth crash. The existence of the postcursor mode(s) also support this theory [26]. There have been results on the diffusion rate of impurities during the crash, which seemed to be in contradiction with the stochastic model [27]. Recent 2D ECEI results have explained these observations [23, 28]: the sawtooth crash occurs in a random localised spot. With the existence of a local crash zone instead of a global one, the measured impurity diffusion rates can be fit in the stochastic model.

The exact generation mechanism of such local crash zone, however, is yet unknown. Recent results on ASDEX Upgrade [29, 30, 31] and HT-7 [32] showed, that a low frequency signal component is visible on the central SXR signals, and it gains energy just before the sawtooth crash. Observations of a second, lower amplitude and lower frequency $n=1$ mode have also been reported on JET [33]. The measured ratio of the frequency of this Low Frequency Sawtooth Precursor (LFSP) and the $(1,1)$ mode - within the uncertainty of the measurements - is close to the conjugate golden mean $G=(\sqrt{5}-1) / 2 \simeq 0.618$. The coupling of two signal components with any incommensurable frequency ratio (not only the golden mean) leads to stochasticity. The golden mean frequency ratio means that the transition from quasi-periodicity to chaos occurs in the energetically most favourable way $[29,34]$. The existence and behaviour of this low frequency mode fits well into the stochastic model, and can be a key element in the understanding of the crash mechanism. This article focuses on the detailed analysis of the data from the central soft X-ray channels of ASDEX Upgrade [35] in order to better understand the behaviour of this component, and the connection between it and other sawtooth precursor modes.

The paper is organized as follows. Section 2 covers the analysis of the time-frequency behaviour of the LFSP. The interaction of the LFSP and the $(1,1)$ mode is investigated in section 3 by bandpower-correlation and bicoherence analysis. The spatial structure of the LFSP was determined from SXR measurements as described in section 4, followed by the discussion of the results in section 5 .

\section{Time-frequency evolution of the LFSP}

The main sawtooth oscillation precursor mode is characterized by an $(m, n)=(1,1)$ spatial structure [18]. Usually one or two of the upper harmonics (the $(2,2)$ and $(3,3)$ mode) also appear before the crash. The interaction of the $(1,1)$ mode and the LFSP can cause a quasi-periodic transition to chaos, which can play a key role in the sawtooth crash [29]. In order to get a quasi-periodic signal followed by a transition to chaos any irrational frequency ratio is sufficient. It is impossible to tell whether a measured ratio is rational or irrational, however the approximated value of the frequency ratio can be estimated. For this reason we have analysed a large number of different sawtooth crashes (20-100 depending on the type of analysis). Various shots have been selected from the shotnumber 
range of \#20975 to \#24006, in accordance with the following constraints. We required shots where the amplification of SXR channels was optimized for the core measurements. The enhanced toroidal rotation due to NBI heating increases the measured frequency of the modes and thus relieves the separation of the $(1,1)$ and LFSP modes in frequency. Statistical analysis demanded cases with relatively long quasistationary precursor activity. The selected shots will be presented later in table 1 .

Most of the analysis tools used in this paper are based on linear continuous timefrequency transformations such as the Short Time Fourier Transformation (STFT) and the Continuous Wavelet Transformation (CWT) [36]. Continuous transformations have the advantage of being time-shift invariant that is crucial in transient signal analysis. By using linear transformations we are able to calculate the power distribution of the signal over the time-frequency plane called the spectrogram (in case of using STFT). In every investigated case the LFSP component was visible on the spectrograms already 20-40 ms before the sawtooth crash. Two examples are shown on figure 1.
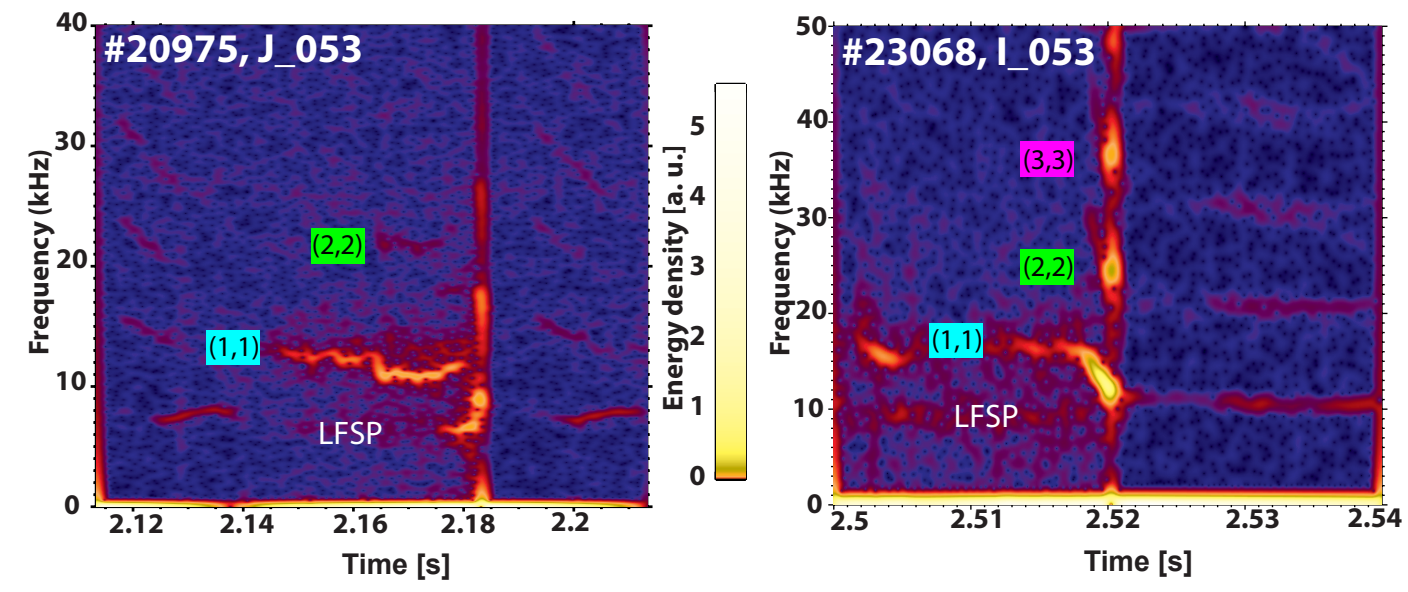

Figure 1. Example spectrograms of different type of sawtooth crashes. The leading $(1,1)$ mode and its harmonics are clearly visible before the crash. An other signal component, identified as the LFSP appears with a frequency lower than the $(1,1)$ mode.

We have noticed during the investigation of the spectrograms that the crashes can be divided into several groups within one shot according to their time-frequency behaviour. Usually five to fifteen consecutive sawtooth crashes have almost identical precursor evolution. The similarity is judged from the frequency of the precursor modes, how early they appear before the crash, and their complex time-frequency evolution. This kind of grouping shows good agreement with the change of the crash frequency within the shot. Two examples are show on figure 2, where point types mark the grouping based on spectrograms. Crashes in the same group either correspond to crashes with the same crash period or to crashes with the same "gradient" of the crash period (marked with diamonds $(\diamond)$ on figure 2). In order to investigate the behaviour of this low frequency component, the event statistics had to be improved. This can be done with averaging over similarly behaving crashes. Every case when we apply averaging of several crashes, that is based on this grouping technique. 

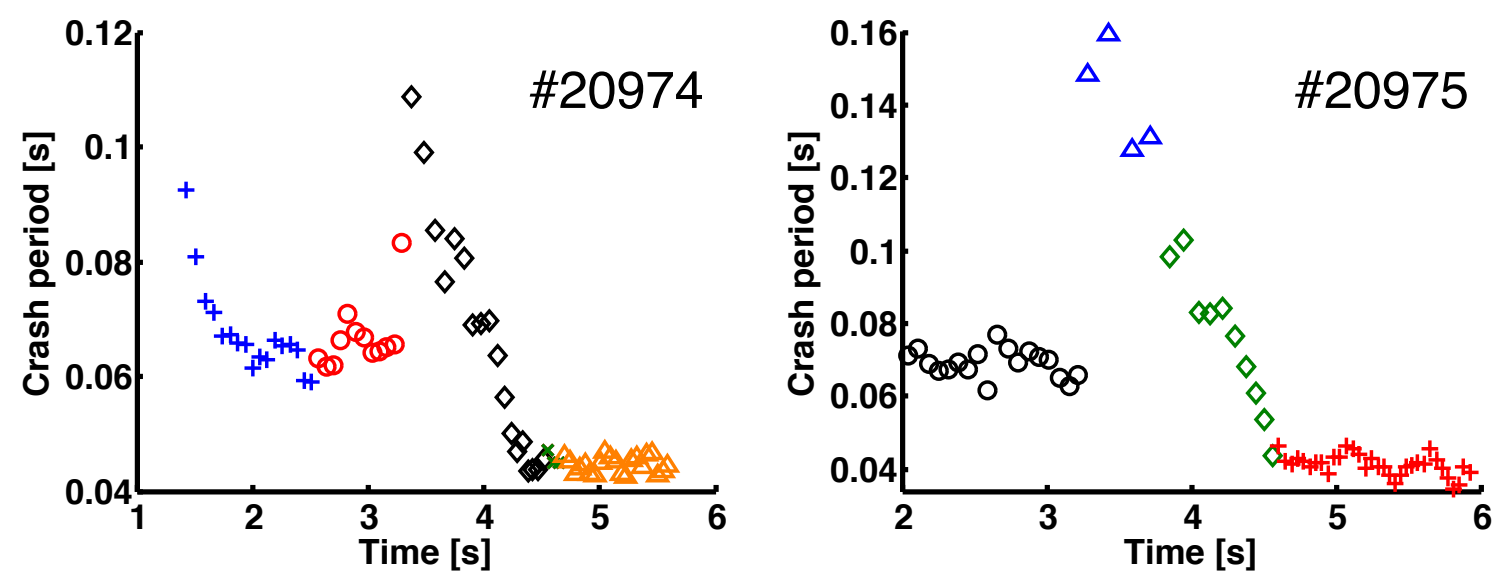

Figure 2. Sawtooth period as a function of time for two shots. Good agreement with the characteristic time-frequency behaviour, marked with different point types.

Shot \#20974 and \#20975 presented on figure 2 were sawtooth control shots, testing the possibility of sawtooth control with ECRH local heating and current drive. This was responsible for the changes in the sawtooth period. ECRH deposition position was changed by $B_{T}$ ramp up. Although the time-frequency dynamics of the precursors have changed due to the different heating, the LFSP was observable in all phases of the control shots. This shows that existence of the LFSP does not depend on the changes of local shear and thus on the sawtooth period.

The LFSP was investigated during different heating- and active sawtooth control schemes (NBI, NBI+ECRH, NBI+ICRH, see table 1). Investigation in the absence of NBI was not possible due to the constraints explained before. The LFSP was observable in all cases with a similar time-frequency structure. The fact that the main phenomena are the same during different shots from different campaigns from year to year as well as with different heating schemes supports the generality of the observed behaviour.

\subsection{Frequency evolution of the LFSP}

To obtain the frequency modulation of a signal, we have to find the local maxima of the mode energy on the time-frequency plane. The easiest way is to select the local maxima of the spectrogram at each time point in a given frequency range, but this is effective only if the investigated mode has an energy significantly larger than that of the background noise. If this condition does not hold, the maximum of the spectrogram at a given time might not belong to the investigated mode. For this reason a new tool had to be developed to estimate the frequency evolution of low energy modes such as the LFSP.

We use a global ridge following algorithm based on graph theory, inspired by $\mathrm{P}$. Varela [37]. The algorithm works as follows. First we create a graph from the spectrogram points, in which each time-frequency point is represented by a node in the graph: $x_{i, j}$, where $i$ stands for time, $j$ stands for frequency index. Edges connect each node at a given 
time point with all the nodes in the following time point: $x_{i, j}$ with $x_{k, j+1}, k=1 \ldots N$ for all $i, j$. In the next step a weight is assigned to each edge, which is estimated using two factors. The first factor represents the search for the ridge of the spectrogram: the larger the amplitude, the smaller the cost associated with the edge leading to the node. The second factor represents that the frequency of the investigated mode shall not change too rapidly: the greater the distance, the greater the cost. The weight of these two factors with respect to each other depends on the signal-to-noise ratio of the given mode we intend to follow. The control parameters of the ridge following algorithm were tested and optimized for the actual signal-to-noise ratios we have in these cases. The shortest path in the weighted graph between the first and last column of the nodes has to be found, this will represent the frequency modulation of the investigated mode, that is larger than the frequency resolution of the spectrogram. We use a modified version of Dijkstra's shortest path algorithm [38] to solve the problem. The original algorithm can be speeded up if we use the special properties of the spectrogram-based graph.
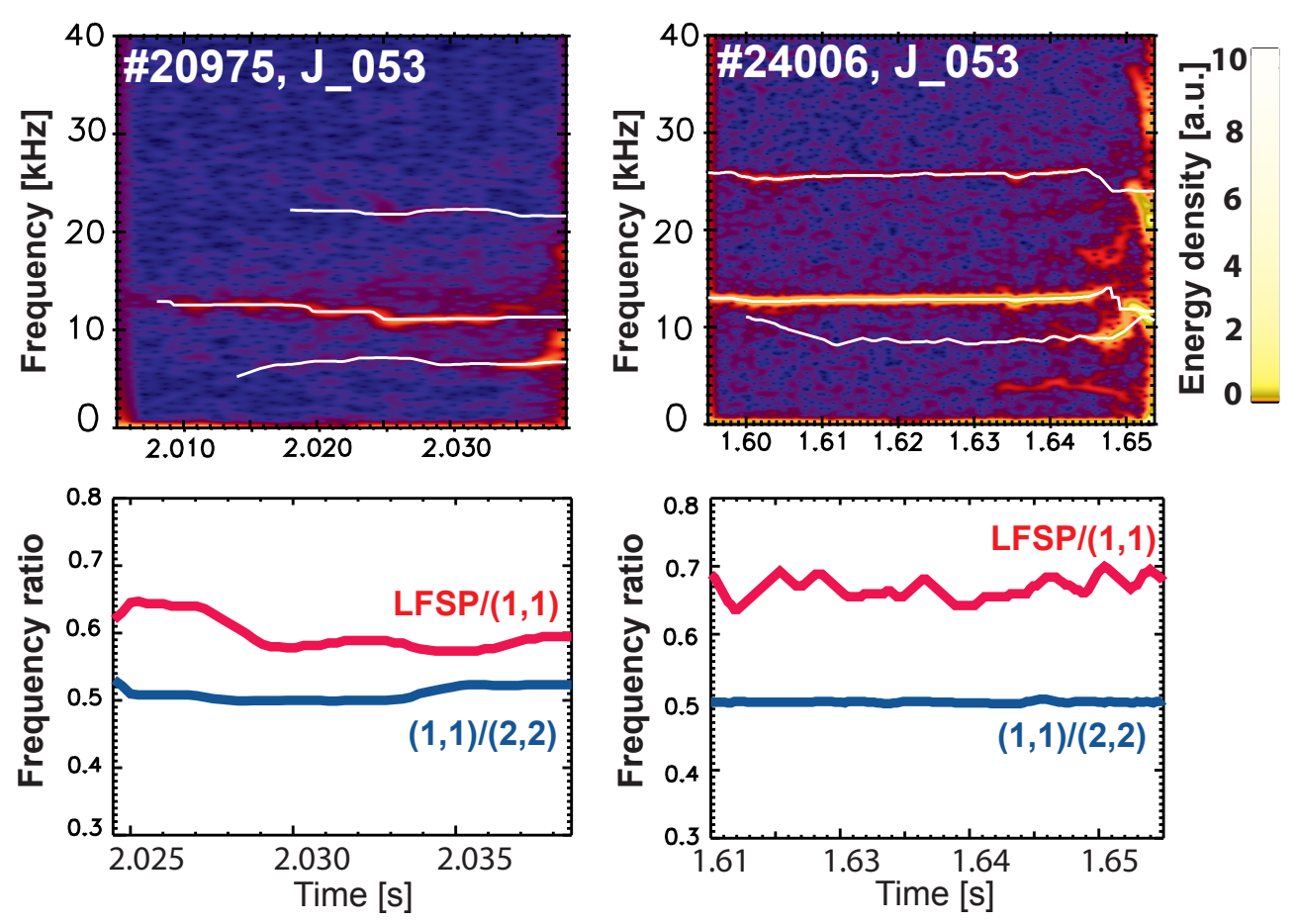

Figure 3. Top: example spectrograms of the sawtooth crash, frequency modulation of the modes overplotted with white. Bottom: Frequency ratios of the different modes as a function of time.

The frequency ratio of the modes as a function of time have been investigated with the global ridge following algorithm. Figure 3 show visualisations of the estimated spectrogram ridges as well as the frequency ratios of the modes as a function of time for two selected crashes.

The frequency ratios were averaged within one shot for the several sawtooth crashes within the selected crash group (see table 2). The main plasma parameters and the observed mean $f_{\mathrm{LFSP}} / f_{(1,1)}$ ratio of the investigated shots are shown in table 1 . Error bars 
are governed by the variation of the frequency ratio in time.

\begin{tabular}{|c|c|c|c|c|c|}
\hline Shot & Heating $[\mathrm{MW}]$ & $B_{T}[\mathrm{~T}]$ & $n_{e}\left[10^{19} / m^{3}\right]$ & $I_{P}[\mathrm{MA}]$ & $f_{\mathrm{LFSP}} / f_{(1,1)}$ \\
\hline 20974 & $\mathrm{NBI}[5.16]+\mathrm{ECRH}[0.35]$ & -2.363 & 6.28 & 0.800 & $0.653 \pm 0.121$ \\
\hline 20975 & $\mathrm{NBI}[5.14]+\mathrm{ECRH}[0.41]$ & -2.379 & 6.32 & 0.801 & $0.611 \pm 0.175$ \\
\hline 22036 & $\mathrm{NBI}[5.06]$ & -2.165 & 6.81 & 0.800 & $0.545 \pm 0.195$ \\
\hline 23068 & $\mathrm{NBI}[10.13]$ & -2.482 & 6.91 & 0.801 & $0.515 \pm 0.187$ \\
\hline 23476 & $\mathrm{NBI}[5.11]+\mathrm{ICRH}[4.96]$ & -2.487 & 9.18 & 1.001 & $0.51 \pm 0.1$ \\
\hline 24006 & $\mathrm{NBI}[5.13]+\mathrm{ICRH}[2.73]$ & -2.454 & 8.71 & 1.003 & $0.65 \pm 0.1$ \\
\hline
\end{tabular}

Table 1. Plasma parameters and observed $f_{\mathrm{LFSP}} / f_{(1,1)}$ ratios of the investigated shots.

As a benchmark of the method we checked the frequency ratio of the $(1,1)$ and the $(2,2)$ modes, and it was $1 / 2$ in all cases. This result supports the reliability of the frequency following algorithm. Although there are some uncertainties, it seems to be clear that the $\operatorname{LFSP} /(1,1)$ ratio is not a low order rational, and it is between 0.5-0.7 in all the investigated cases.

\subsection{Power evolution of the LFSP}

The appearance of the low frequency component is usually visible several tens of milliseconds before the crash, but the energy of the component is very low during most of the precursor phase. The low-energy phase lasts for 10-40 ms and is followed by a swift energy gain $\sim 5 \mathrm{~ms}$ before the sawtooth crash, as observed on the spectrograms (figure 1). We can get a better estimate if we investigate the evolution of the bandpower of the LFSP during the whole crash period. The bandpower is estimated by integrating the spectrogram in frequency over a certain frequency range [39].

To be able to quantitatively characterize the energy evolution we have fitted exponential curves $\left(P=A \cdot \exp \left\{\gamma_{P} \cdot t\right\}+B\right)$ to the bandpowers of the LFSP for the investigated sawtooth crashes in the precursor phase. Here $A$ is the bandpower magnitude, $B$ is the background noise level of the given frequency band, and $\gamma_{P}$ can be understood as the growth rate of the bandpower of the LFSP before the crash. The fit region was selected to end at least $0.8 \mathrm{~ms}$ before the bandpowers reach their maxima. $0.8 \mathrm{~ms}$ is the half width of the Gaussian window that was used during the estimation of the bandpowers and hence a good estimate of the standard deviation of the power peak. The time axes were shifted so that the crash occurs at 0 . We have to note that the LFSP bandpower usually saturates $\sim 2 \mathrm{~ms}$ before the sawtooth crash, and sometimes even shows a local minimum $\sim 1 \mathrm{~ms}$ before the crash event itself. An example bandpower with a fit is shown on figure 4.

We have calculated the weighted average of the fitted parameters in several crash groups containing 8-17 crashes each, presented in table 2. The uncertainty of the 


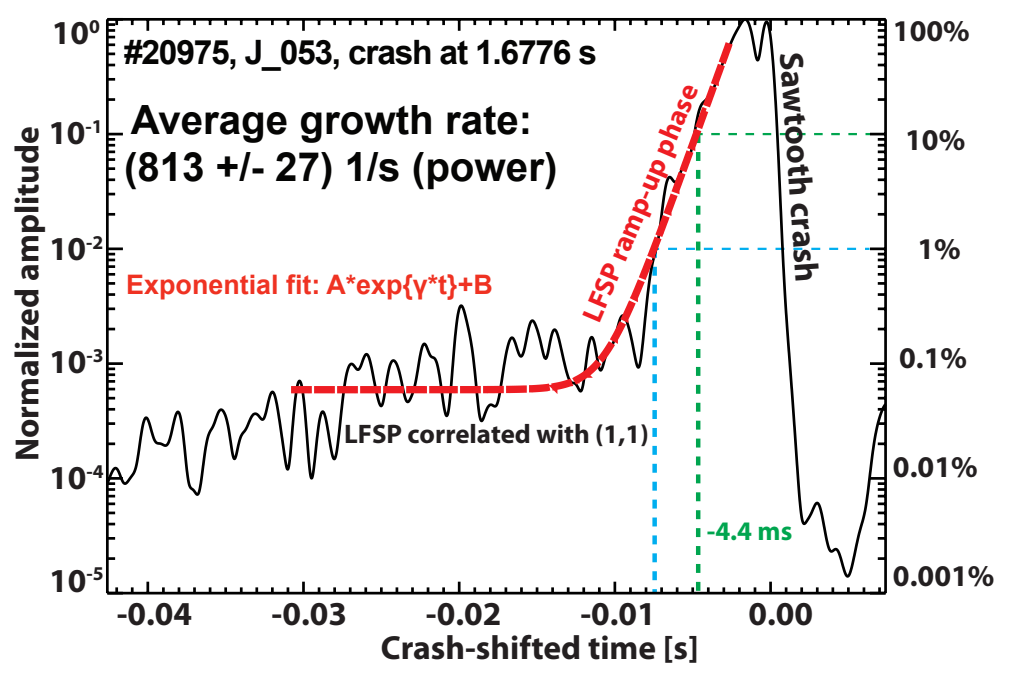

Figure 4. Example exponential fit (dashed line) of the bandpower of the LFSP. The measured amplitude growth rate is $\gamma_{A}=(4071 / \mathrm{s} \pm 3 \%)$, much smaller than the Alfvén growth rate of $1.5-2 \cdot 10^{5} 1 / \mathrm{s}$.

parameters is low, they show good agreement between the different shots, consequently the ramp-up of the low frequency component is very uniform from the amplitude growth point of view. The average power growth rate is $\gamma_{P}=(8131 / \mathrm{s} \pm 3 \%)$, so the amplitude growth rate is $\gamma_{A}=(4071 / \mathrm{s} \pm 3 \%)$. The component in average reaches $10 \%$ of its maximal power $\tau_{10 \%}=(-4.4 \mathrm{~ms} \pm 13 \%)$ before the crash. Although a normalized figure was presented, the absolute value of the LFSP bandpower before the crash also has a rather uniform value. These observations can be the basis to extract the critical values of the underlying parameters such as LFSP amplitude necessary for the crash.

\section{Interaction of the $(1,1)$ and LFSP modes}

The interaction of the $(1,1)$ and the LFSP may play a key role in the mechanism of the sawtooth crash [29]. However, the origin of the mode and the source of its energy is not yet discovered.

\subsection{Bandpower-correlation analysis}

One way to characterize the interaction of different modes is the bandpower-correlation method [39]. Our early results have shown a correlation between the bandpowers of the $(1,1)$ and the LFSP [30]. In order to extend this investigation we had to improve event statistics. This could be achieved with averaging the bandpower correlation functions for several crashes with similar behaviour, as described in section 2 .

We must avoid the deterministic energy growth in the energy of the modes right before the crash, thus the selected time window for the correlation estimation was 
terminated at least $1.6 \mathrm{~ms}$ before the crash, where $1.6 \mathrm{~ms}$ is the width of the Gaussian window that was used during the estimation of the bandpowers. The exact time windows have been selected manually for each crash using spectrograms. This allows a better localization of the quasistationary period compared to an automatic selection that has to be conservative. We calculated the bandpower cross-correlation functions between the LFSP and the $(1,1)$ for these time windows and averaged the results for each crash group. Figure 5a shows an example of the bandpower correlation functions. The $95 \%$ confidence interval (marked with two dashed lines) was calculated from the standard deviation of the averaging.
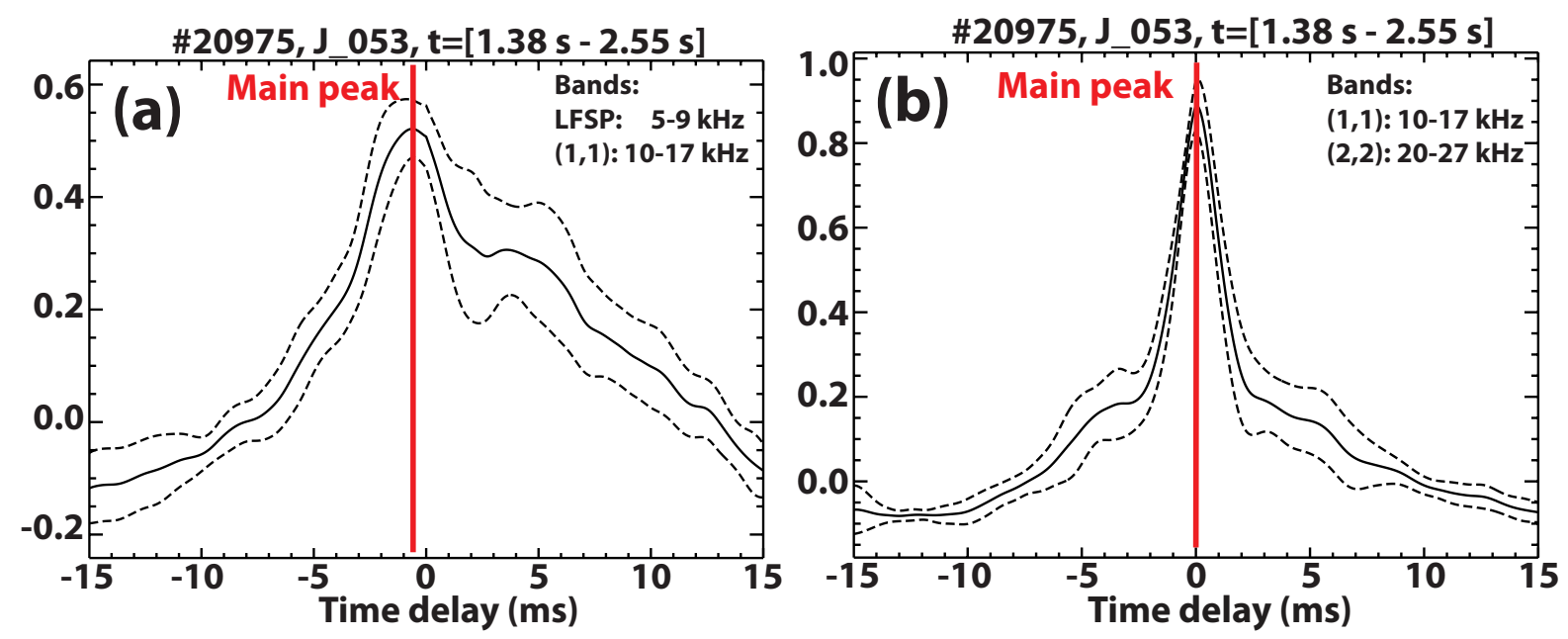

Figure 5. (a) Averaged bandpower cross-correlation between the LFSP and the $(1,1)$ mode. (b) Averaged bandpower cross-correlation between the $(1,1)$ and $(2,2)$ modes.

\begin{tabular}{|c|c|c|c|c|c|c|}
\hline Shot & Time win. & $f_{2}[\mathrm{kHz}]$ & $f_{(1,1)}[\mathrm{kHz}]$ & Averages & $\tau[\mathrm{ms}]$ & $\mathrm{C}[\%]$ \\
\hline $20974 \mathrm{~A}$ & $25 \mathrm{~ms}$ & $4-9$ & $10-16$ & 17 & $-1 \pm 0.8$ & $45 \pm 10$ \\
\hline $20975 \mathrm{D}$ & $30 \mathrm{~ms}$ & $4-7$ & $8-15$ & 15 & $-1 \pm 0.8$ & $50 \pm 5$ \\
\hline $20975 \mathrm{~A}$ & $40 \mathrm{~ms}$ & $5-9$ & $10-17$ & 8 & $-1 \pm 0.8$ & $40 \pm 10$ \\
\hline 22036 & $40 \mathrm{~ms}$ & $7-13$ & $15-20$ & 1 & $-1.7 \pm 0.5$ & $45 \pm 15$ \\
\hline 23068 & $40 \mathrm{~ms}$ & $6-11$ & $13-20$ & 1 & $-1.4 \pm 0.5$ & $75 \pm 11$ \\
\hline 24006 & $35 \mathrm{~ms}$ & $8-10.5$ & $12-15$ & 5 & $0 \pm 0.8$ & $55 \pm 20$ \\
\hline
\end{tabular}

Table 2. Bandpower cross-correlation analysis for different crash groups. The table shows the length of the time window, the frequency ranges, the number of averages, the correlation value $(\mathrm{C})$ and delay $(\tau)$.

On figure 5a the magnitude of the larger peak of the CCF is $(55 \pm 5) \%$ at the negative time delay of $(-1 \pm 0.8) \mathrm{ms}$ : the LFSP is delayed compared to $(1,1)$. The assymmetry of the correlation functions is also a characteristic feature. Table 2 contains the main parameters of the averaged bandpower correlation functions for each group. The table also contains two non-averaged bandpower-correlations from shot 22036 and 23068. The 
correlation of the $(1,1)$ mode and the low frequency component is systematic, the time lag varies between 0 and -2 ms within the uncertainty of the measurements.

Figure 5b shows a typical example of an averaged bandpower correlation function between the modes $(1,1)$ and $(2,2)$. This peak is symmetric with an amplitude of $(90 \pm 5) \%$ and a time delay of 0 , which is a completely different structure than in the case in figure $5 \mathrm{a}$. This result implies that the LFSP and the $(1,1)$ are not harmonics and not coupled with having some small-denominator rational frequency ratio. The very high correlation with zero time delay between $(1,1)$ and $(2,2)$ modes suggests that the appearance of the higher harmonics is due to the anharmonicity of the $(1,1)$ mode.

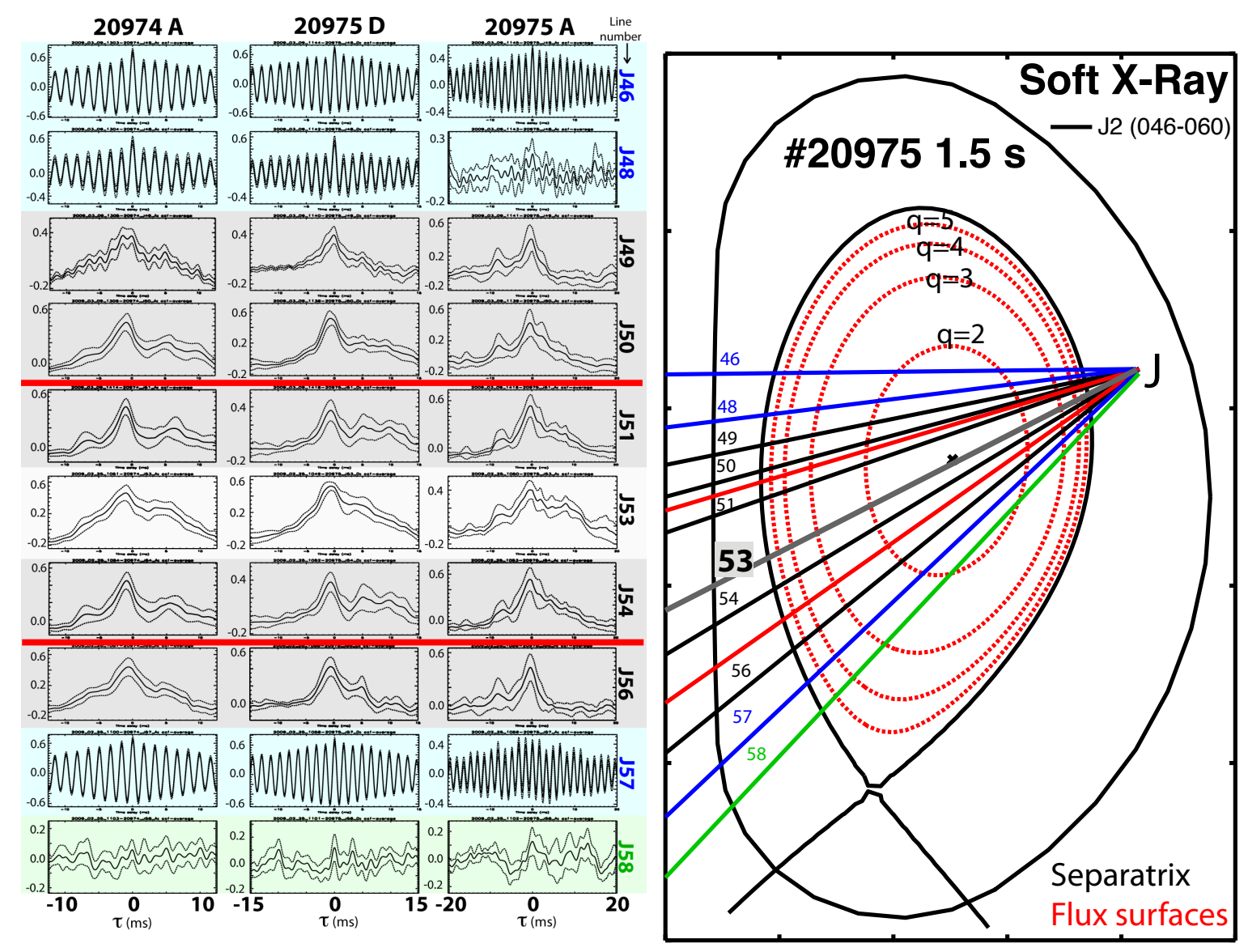

Figure 6. (a): Spatial dependence of the correlation functions for 3 different cases. (b) Spatial distribution of SXR channels in AUG. Colors indicate the corresponding CCFs on (a). Red line marks the inversion radius.

The correlation functions also show a clear spatial dependence, as presented on figure 6. In channels J49-J56 we see the same pattern that was observed in the core plasma. For outer channels the correlation function shows a periodic behaviour with 1.5 ms period, which is a result of an electronic noise caused by the flywheel generator [40]. It shows clearly that in this spatial region no other correlated plasma phenomenon is present, hence the noise can dominate the correlation functions. Figure 6.b shows this spatial dependence in the poloidal cross-section of the ASDEX Upgrade tokamak. The two 
red lines mark the inversion radius. Black channels mark the correlation pattern, which appears outside the inversion radius as well. Blue refers to channels where the CCFs are dominated by the flywheel noise, and the green channel marks the vanishing point of this periodic pattern. The point where the generator noise is overcome by signal components originated from the plasma is the $q=2$ surface, where we expect the existence of $m / n=2$ modes. The spatial dependence of the correlation functions give a rough estimate for the spatial localization of the interaction of the LFSP and the $(1,1)$ mode, that appears to be visible slightly outside the inversion radius. This suggests that the modes are also present outside the inversion radius. Some experiments claim that the $q=1$ surface lies outside the sawtooth inversion radius [41, 42] and it is suggested by theory as well [43]. The $(1,1)$ mode is associated with the $q=1$ surface and it almost always have a small perturbations outside the $q=1$ surface. Our observations coincide with the possibility that the inversion radius may be somewhat smaller than the radius of the $q=1$ surface.

\subsection{Bicoherence analysis}

By means of bicoherence analysis, we are able to measure phase coupling between signal components [44]. Phase coupling occurs in that case, when two frequencies and their sum frequency are present at the same time in a signal, and their phases satisfy the $\varphi_{1}+\varphi_{2}=\varphi_{3}+$ const. equation. The result of the bicoherence calculation is a matrix, which might be plotted as a function of two frequency variables. If the signal contains components at $f_{1}, f_{2}$ and $f_{3}=f_{1}+f_{2}$ frequencies, and the phases are coupled, the bicoherence take on a value close to 1 at the $\left(f_{1}, f_{2}\right)$ point, without reference to the modes' amplitude. However, the absence of phase coupling between the components result a value near to 0 . If the signal contains a harmonic and its upper harmonic at $f$ and $2 \cdot f$ frequency, the bicoherence is close to 1 at the $(f, f)$ point.

The bicoherence plot shown on figure 7 was calculated for two different time intervals for the crash at $t=1.6537 \mathrm{~s}$ in shot 24006 . In the first case, shown on figure $7 \mathrm{a}$, the time window of the bicoherence calculation was [1.61,1.64], ending $15 \mathrm{~ms}$ before the crash, well before the ramp-up phase of the LFSP. Despite the low energy of the LFSP that is barely visible on the autospectrum, there is a significant 0.6 bicoherence between the $(1,1)$ and the LFSP. This suggests the existence of a nonlinear interaction between the two modes already in the low-energy phase of the LFSP, in agreement with the bandpower-correlation

analysis. In the second case, shown on figure $7 \mathrm{~b}$, the time window was $[1.61,1.65]$, ending only $5 \mathrm{~ms}$ before the crash, in the early ramp-up phase of the LFSP. The bicoherence rises slightly between the $(1,1)$ and the LFSP modes. The bicoherence also shows the existence of the upper harmonic of the LFSP, which was not visible on the spectrograms. The nonlinear coupling between the $(1,1)$ and the $(2,2)$ mode is clear in both cases. 

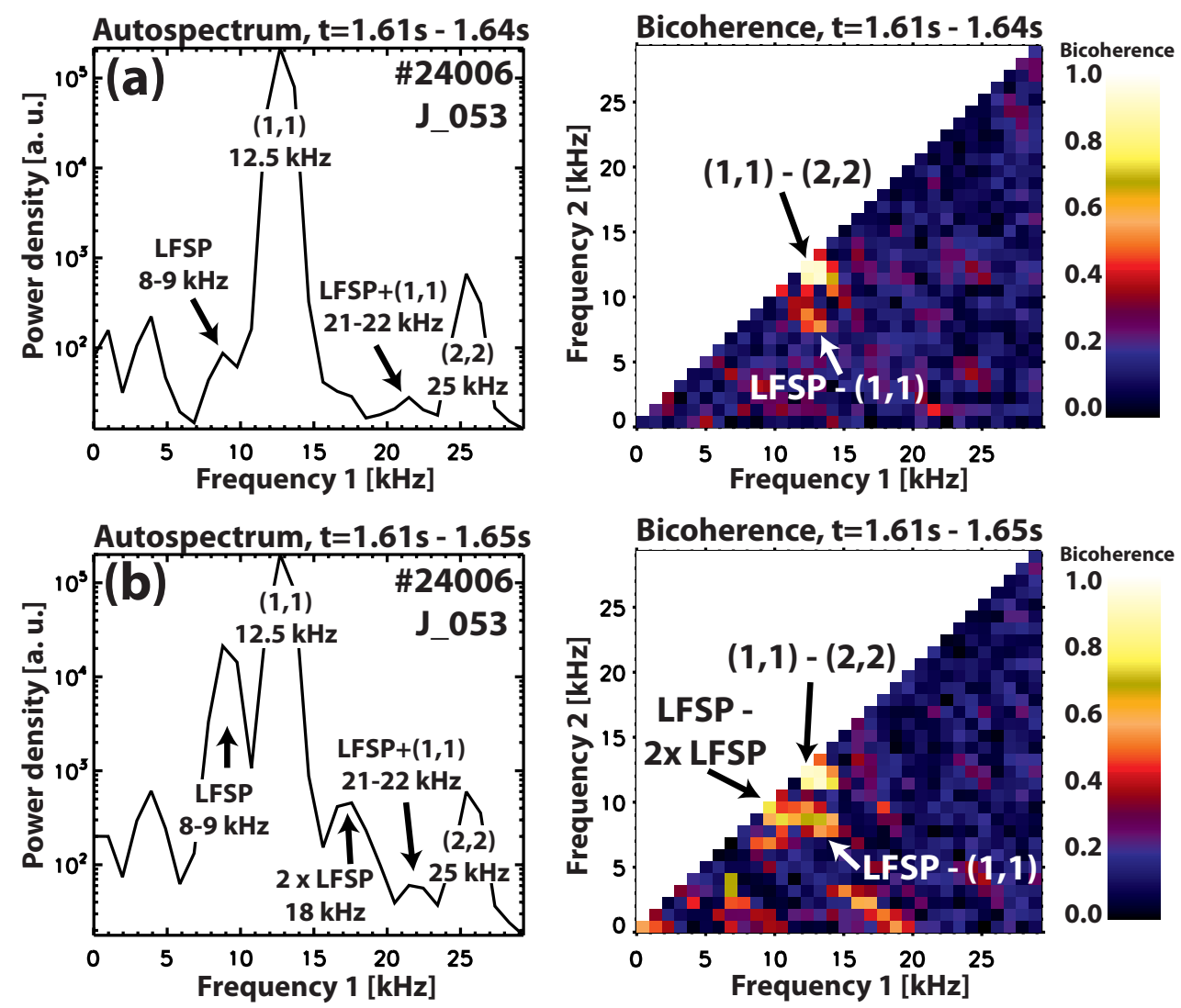

Figure 7. Autospectrum and bicoherence calculated for two different time intervals ((a) $\mathrm{t}=1.61 \mathrm{~s}-1.64 \mathrm{~s}$ and $(\mathrm{b}) \mathrm{t}=1.61 \mathrm{~s}-1.65 \mathrm{~s})$ for the crash in \#24006 at $1.6537 \mathrm{~s}$.

\section{Spatial structure}

The spatial structure of the LFSP is a key issue in understanding the phenomenon. However, the transient nature of the mode and its localization in the plasma core makes it very difficult to detect it and to estimate the mode numbers. The required time resolution can be achieved with only a limited number of diagnostics. Magnetic signals are the most preferred for mode number calculations, but it is not feasible for low energy transient core modes. For this reason, we have applied wavelet based methods for detecting shortlived plasma eigenmodes and determining their spatial structure $[45,46]$ on soft X-ray measurements.

The wavelet minimum coherence method can safely detect low amplitude coherent modes at the expense of only a small loss in temporal resolution by synthetising information of all available signals. Wavelet coherence is calculated by the formula

$$
C O H_{x, y}(u, \xi)=\frac{\left|\left\langle W x(u, \xi) W^{*} y(u, \xi)\right\rangle\right|}{\sqrt{\left\langle|W x(u, \xi)|^{2}\right\rangle\left\langle|W y(u, \xi)|^{2}\right\rangle}},
$$

where $W x(u, \xi)$ and $W y(u, \xi)$ are the continuous wavelet transforms of signals $x$ and $y$ using analytical wavelets (Morlet wavelet [36] in the present paper), $*$ denotes 
the complex conjugate, $u$ and $\xi$ are the time and frequency parameters respectively, and $\langle\ldots\rangle$ denotes the averaging implemented by convolution smoothing with an affine invariant rectangular kernel. This way, invariance properties of the continuous wavelet transform - essential for processing transient signals - are preserved in wavelet coherence [45]. Spatially extended coherent structures in the plasma are in most cases picked up by several probes. Coherence can be calculated for each pair, and coherence information can be combined by calculating the wavelet minimum coherence [45].

The mode number determination method presented in this paper is based on the phase of the continuous analytical wavelet transform:

$$
\vartheta_{x, y}(u, \xi)=\arg \left\{\left\langle W x(u, \xi) W^{*} y(u, \xi)\right\rangle\right\}, \quad \arg (z)=\operatorname{arctg} \frac{\operatorname{Im} z}{\operatorname{Re} z}
$$

For each $(u, \xi)$ point of the time-frequency plane, $\vartheta_{x, y}(u, \xi)$ relative phases between all $(x, y)$ pairs of signals are calculated. For a pure sinusoidal structure, these relative phases would lie on a straight line as a function of the $\phi_{x, y}$ relative probe position. The method has been tested for both toroidal and poloidal mode numbers, with the right choice of $\phi_{x, y}$ (i.e. use a straight field line coordinate system at a given radial location) as will be described later. The slope of the best fitting straight line gives the mode number with the residual defined as:

$$
\mathcal{Q}_{l}(u, \xi)=\sum_{x, y}\left\|\vartheta_{x, y}(u, \xi)-l \cdot \phi_{x, y}\right\|_{2 \pi}^{2}
$$

where $l=\{m, n\}$ is the toroidal or poloidal mode number and $\|\ldots\|_{2 \pi}$ is the norm by taking the optimum shift of $\Theta_{x, y}$ by $z \cdot 2 \pi, z \in \mathbb{Z}$. Smoothing in this method is optional, but largely improves the accuracy, and also enables the calculation of wavelet minimum coherence. This method gives a most fitting mode number for each point on the time-frequency plane. However, mode numbers are a relevant quantity only in limited regions, where coherent modes exist. We can find these regions based on a criterion for the $\min _{l}\left\{\mathcal{Q}_{l}(u, \xi)\right\}$ values, or on wavelet minimum coherence, or on the combination of both, as in this paper.

Toroidal mode numbers were estimated using two identical SXR cameras (F and G) placed $135^{\circ}$ apart toroidaly but having the same lines of sight in the poloidal cross-section. Up to 4 central channel pairs inside the sawtooth inversion radius (shown on figure 8a) were used for mode number estimation with the averaging of 3 consecutive measurements.

For the poloidal mode numbers we had to select lines of sight in the same toroidal cross-section that are tangential to approximately the same flux surface and measure high bandpower values on the mode frequency. An example is shown on figure 8b.

Due to toroidal effects and the gradient of the magnetic field, the mode structure is distorted. This can be compensated if we transform the inhomogeneous magnetic field to a homogeneous one by using a straight field line $\Theta^{*}$ poloidal angle coordinate [47] instead of the geometrical $\Theta$ coordinate shown on figure 8c. 

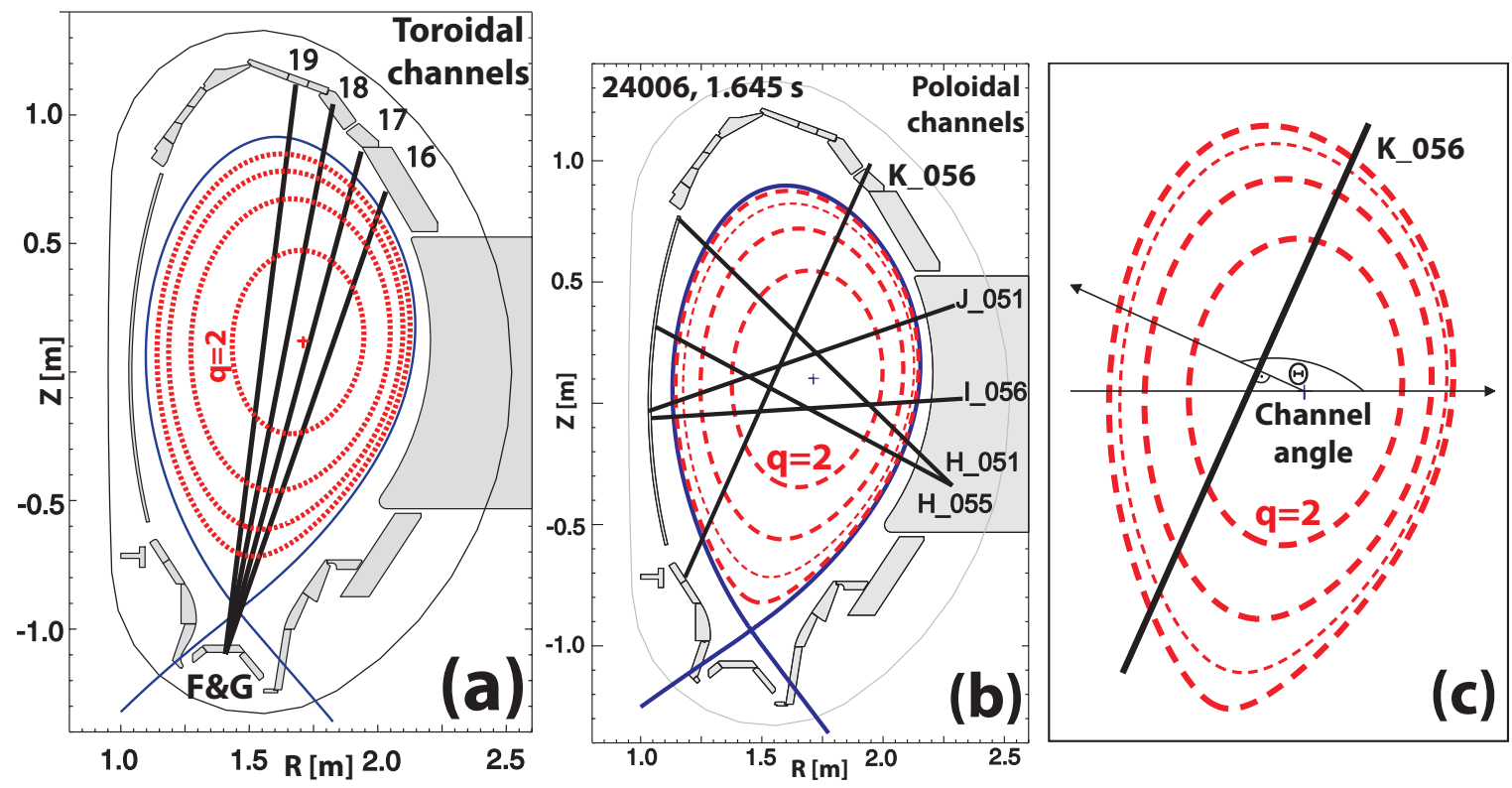

Figure 8. Example of soft X-ray channels selected for (a) toroidal, (b) poloidal mode number determination. (c): Geometrical angle $(\Theta)$ associated with a given line of sight.
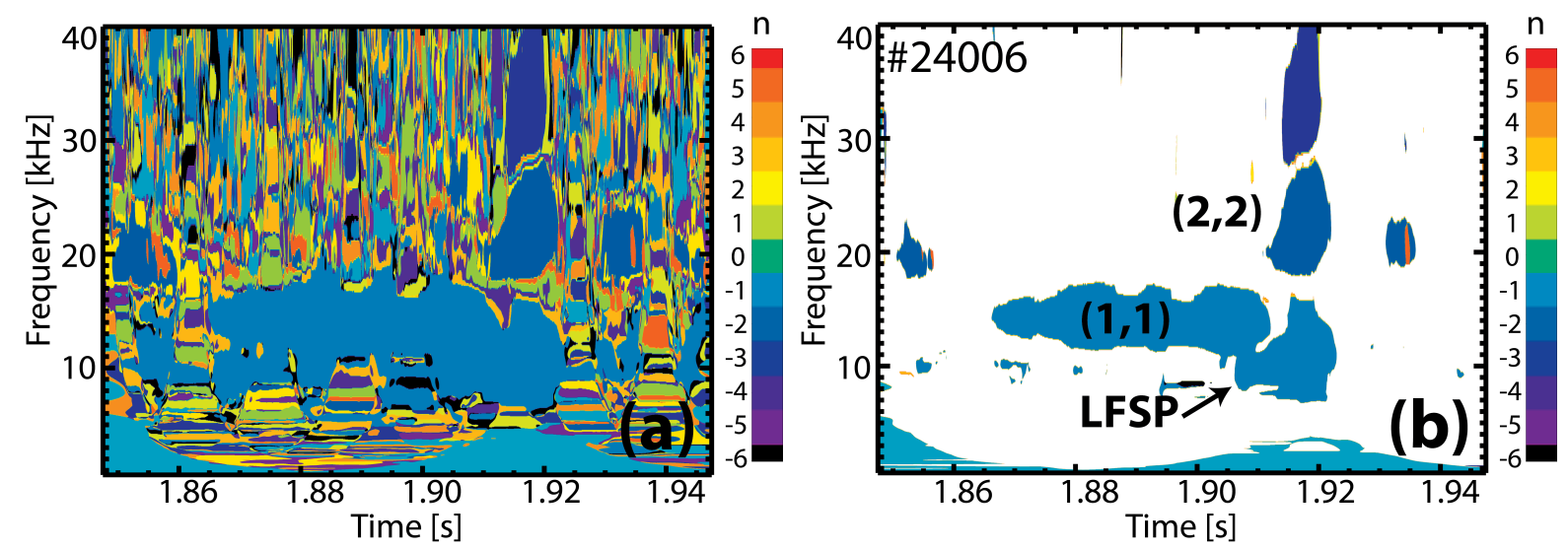

Figure 9. (a) Toroidal mode numbers of the sawtooth precursors calculated from SXR measurements. (b) Coherence and min- $\mathcal{Q}$ filtered mode numbers. The $(1,1)$ and harmonics are clearly observable. The toroidal mode number of the LFSP is $n=1$.

According to the measurements shown on figure 9 and figure 10, the mode number of the LFSP is $(1,1)$. The sign of the mode numbers mark the direction of propagation with respect to the coordinate system chosen for the effective probe angles. In this case the mode propagation is in the ion diamagnetic drift direction. 

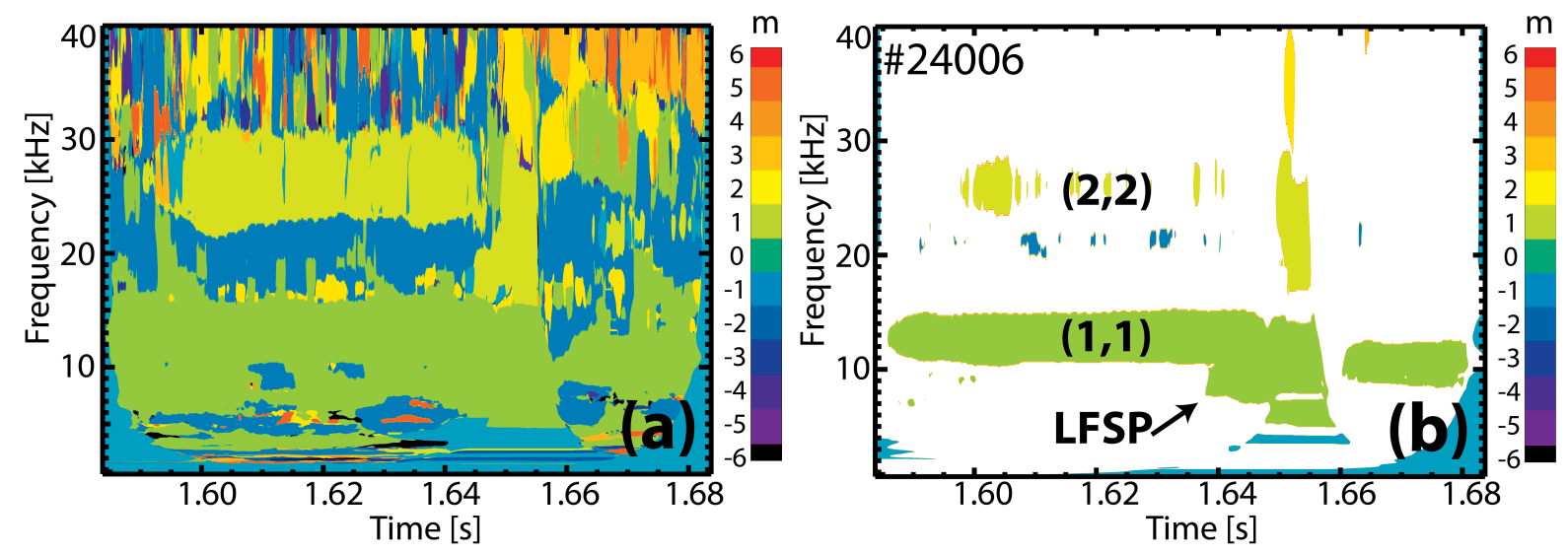

Figure 10. (a) Poloidal mode numbers of the sawtooth precursors calculated from SXR measurements. (b) Coherence and min- $\mathcal{Q}$ filtered mode numbers. The $(1,1)$ and harmonics are clearly observable. The poloidal mode number of the LFSP is $m=1$.

\section{Discussion}

One of the most important aims of this research connected to the sawtooth oscillation is to clarify the sawtooth crash mechanism. In this paper we have presented several new pieces of information on the main modes involved in the crash. Our paper aims to complement the work of theoretical modellers and concentrates mainly on presenting clear experimental evidence together with presenting a number of new data analysis methods.

The LFSP was observed and analysed just before the sawtooth crashes on ASDEX Upgrade. It is possible that the interaction of the LFSP and the $(1,1)$ internal kink mode is associated with the sawtooth crash [29]. The estimated frequency ratio of the two modes was found to be between 0.5 and 0.7 in the investigated cases. This is close to the theoretically most favourable value $\left(f_{\mathrm{LFSP}} / f_{(1,1)} \simeq 0.618[34]\right)$ within the uncertainty of the measurements. Our knowledge on the LFSP and the interaction mechanism is still not sufficient for a clear theoretical understanding, nonetheless, we present a few possible ways how the LFSP can be fit into different sawtooth crash models.

A potential explanation of the experimental results is the following. If the $(1,1)$ kink mode already exists in the plasma, it provides a strong periodic drive force that can excite other modes as well (directly via e.g. magnetic coupling; or indirectly through the change of the profiles [48]). The mode numbers of the LFSP was found to be identical to the $(1,1)$ internal kink mode, this also means the LFSP is located at the same radial position, at the $q=1$ surface. In the view of these experimental results, the excitation of the LFSP by the $(1,1)$ is very likely via magnetic coupling. This is possible even if the frequency ratio is irrational. The main frequencies have upper harmonics or sidebands, and the frequency space is filled up in a dense way. If there is a finite $\delta f$ within which two frequencies can synchronize, than the sidebands can interact with each other. If many pairs are synchronized at the same time that is exactly a chaotic stage [34]. A sign of this 
interaction and a possible energy transfer is the measured bandpower-crosscorrelation, and the high value of bicoherence also suggests the existence of a (nonlinear) coupling between the two modes. These latter two results are very improbable in the case of purely separate drive mechanisms. However, the question of the interaction mechanism and the actual drive behind the LFSP has to be addressed with simulations in the future.

Provided that the frequency ratio was not irrational, there is still the possibility that the LFSP plays a role. (Also, magnetic coupling occurs with higher probability if the mode frequencies are rational.) It has been shown that upper harmonics can contribute to the stochastization of the plasma core [21, 22]. Following the same logic, a lower frequency component with commensurate spatial structure can create a relatively broad stochastic layer near the $(1,1)$ island separatrix and especially at the $\mathrm{X}$ point. This coincides with the 2D ECE measurements of the crash phase [49]. The implications of the outlined model on the evolution of the $q$ profile are also consistent with the measurements, namely that the position of the $q=1$ surface is preserved and that $q$ on axis remains below unity. The interaction of the LFSP and the $(1,1)$ kink implies a partial reconnection procedure that is consistent with the observations that heat comes out from the central core region through the X-point of the $(1,1)$ island and the $(1,1)$ island survives the crash [49]. The sudden onset of the crash, the rapidity of the temperature collapse and the incomplete relaxation of the current profile can also be explained by the interaction of modes with commensurate spatial structure [22].

The difference in the observed frequencies is possibly due to the difference of the mode types. The LFSP is most probably a resistive mode according to the growth rate of $\gamma_{A} \sim 4001 / \mathrm{s}$. As described by Hastie et al [50] the resistive growth rate scaled with the Alfvén frequency is $\gamma_{A} / \omega_{A} \leq 2 \cdot 10^{-4}$ for magnetic Reynolds numbers over $S=10^{7}$. In the investigated shots the Alfvén frequency is $4.5-6.5 \cdot 10^{6} 1 / \mathrm{s}$, that gives $\gamma_{A} / \omega_{A}=1.1-1.6 \cdot 10^{-4}<2 \cdot 10^{-4}$, in excellent agreement with resistive theory. The internal kink is often characterized as an ideal mode before the crash [51]. However, an ideal mode cannot be responsible for changes in the magnetic topology - reconnection while a resistive can be [43]. In the ASDEX Upgrade the presence of a $(1,1)$ island is instantly visible after the crash, but not before [49]. The latter experimental observations cannot be described solely with ideal MHD theory.

We understand the LFSP as a secondary instability driven by the $(1,1)$, that causes, or contributes to the crash. During the years, several different crash models have been proposed, each with experimental support [9]. The LFSP can play a role, as outlined above, in the models involving field line stochasticity, chaos or partial magnetic reconnection. There are indications that the sawtooth crash might be governed by different mechanisms in the various devices [52], or by a mixture of the possible mechanisms. One of the latter is the possibility that the aforementioned magnetic reconnection process excites secondary ideal MHD instabilities during the crash [48], that would explain the rapidity of the collapse. However, there is the possibility that the LFSP is excited, driven or destabilized by the changes of the parameter profiles initiated by the $(1,1)$ mode evolution. 
In the latter case the interaction of the reconnection process (that is enhanced by the presence of the LFSP) and the secondary instabilities is very complex and yet unclear. These questions cannot be answered without extensive simulation studies.

Either way, it seems very improbable that a crash model without (partial) magnetic reconnection can be consistent with all the experimental observations [48, 49], and as outlined above, the LFSP might play a crucial role in the reconnection process.

\section{Conclusions}

This paper presents a detailed analysis of the low frequency sawtooth precursor (LFSP) mode appearing at a slightly lower frequency than the $(1,1)$ internal kink mode, as observed on the soft X-ray (SXR) diagnostic of the ASDEX Upgrade tokamak. Indications of a second, lower frequency sawtooth precursor have been reported on JET [33] and HT-7 [23] as well. A number of advanced data analysis methods is also presented, such as the ridge following algorithm, bandpower-correlation, using bicoherence for MHD modes, and a wavelet based method to determine core mode numbers on the time-frequency plane using SXR signals. Most of the applied techniques are new or haven't been applied in such a way before.

The frequency ratios were calculated with an instantaneous frequency following algorithm. The frequency ratio of the two modes does not show any particular temporal development pattern and it is in the 0.5-0.7 range. Analysis of the energy evolution of the mode showed a systematic behaviour, exponential growth with the growth rate of $\gamma_{A} \simeq 4001 / \mathrm{s}$, which was shown to be consistent with the growth rate of a resistive core mode [50].

During the stationary precursor phase well before the sawtooth crash, the energy modulation of the LFSP mode correlates with the energy modulation of the $(1,1)$ internal kink mode. Measured correlation is $50 \%$ in most cases having $1 \mathrm{~ms}$ time delay between the two. This indicates the existence of an interaction between the two modes already 20$40 \mathrm{~ms}$ before the crash event. The correlation functions show a clear spatial dependence, the correlation of the two modes being visible also for lines of sight slightly outside the inversion radius. The high value of the measured bicoherence also suggests the existence of nonlinear coupling between the two modes already $15 \mathrm{~ms}$ before the crash.

The mode structure was determined with a wavelet based method used on SXR measurements [46]. Results indicate that the toroidal and poloidal structure of the LFSP agrees with that of the $(1,1)$ kink precursor mode. The agreement between the spatial structures may enhance possible interactions of the modes. Possible mechanisms of such an interaction leading to the sawtooth crash are presented shortly in the discussion. The LFSP is expected to play a role in the partial magnetic reconnection process, hence every crash model involving such reconnection can be affected by the existence of the LFSP. 


\section{Acknowledgments}

This work, supported by the European Communities under the contract of Association between EURATOM and the Hungarian Academy of Sciences, was carried out within the framework of the European Fusion Development Agreement. The views and opinions expressed herein do not necessarily reflect those of the European Commission. The authors would like to thank A. Gude for useful discussions on the soft X-ray signals.

[1] S. von Goeler, W. Stodiek, and N. Sauthoff. Studies of internal disruptions and $m=1$ oscillations in tokamak discharges with Soft-X-Ray tecniques. Physical Review Letters, 33(20):1201-1203, November 1974. http://prola.aps.org/abstract/PRL/v33/i20/p1201_1.

[2] F Porcelli, D Boucher, and M N Rosenbluth. Model for the sawtooth period and amplitude. Plasma Physics and Controlled Fusion, 38(12):2163, 1996. http://stacks.iop.org/0741-3335/38/i= $12 / \mathrm{a}=010$.

[3] Bo Hu, R. Betti, and J. Manickam. Kinetic stability of the internal kink mode in ITER. Physics of Plasmas, 13(11):112505, 2006. http://link.aip.org/link/?PHP/13/112505/1.

[4] O. Sauter, E. Westerhof, M. L. Mayoral, B. Alper, P. A. Belo, R. J. Buttery, A. Gondhalekar, T. Hellsten, T. C. Hender, D. F. Howell, T. Johnson, P. Lamalle, M. J. Mantsinen, F. Milani, M. F. F. Nave, F. Nguyen, A. L. Pecquet, S. D. Pinches, S. Podda, and J. Rapp. Control of neoclassical tearing modes by sawtooth control. Phys. Rev. Lett., 88(10):105001, Feb 2002. http://prl.aps.org/abstract/PRL/v88/i10/e105001.

[5] A. Gude, S. Gunter, M. Maraschek, H. Zohm, and the ASDEX Upgrade Team. Temporal evolution of neoclassical tearing modes and its effect on confinement reduction in ASDEX Upgrade. Nuclear Fusion, 42(7):833, 2002. http://stacks.iop.org/0029-5515/42/i=7/a=306.

[6] T.C. Hender, J.C Wesley, J. Bialek, A. Bondeson, A.H. Boozer, R.J. Buttery, A. Garofalo, T.P Goodman, R.S. Granetz, Y. Gribov, O. Gruber, M. Gryaznevich, G. Giruzzi, S. Gunter, N. Hayashi, P. Helander, C.C. Hegna, D.F. Howell, D.A. Humphreys, G.T.A. Huysmans, A.W. Hyatt, A. Isayama, S.C. Jardin, Y. Kawano, A. Kellman, C. Kessel, H.R. Koslowski, R.J. La Haye, E. Lazzaro, Y.Q. Liu, V. Lukash, J. Manickam, S. Medvedev, V. Mertens, S.V. Mirnov, Y. Nakamura, G. Navratil, M. Okabayashi, T. Ozeki, R. Paccagnella, G. Pautasso, F. Porcelli, V.D. Pustovitov, V. Riccardo, M. Sato, O. Sauter, M.J. Schaffer, M. Shimada, P. Sonato, E.J. Strait, M. Sugihara, M. Takechi, A.D. Turnbull, E. Westerhof, D.G. Whyte, R. Yoshino, H. Zohm, the ITPA MHD, Disruption, and Magnetic Control Topical Group. Chapter 3: MHD stability, operational limits and disruptions. Nuclear Fusion, 47(6):S128, 2007. http: //stacks.iop.org/0029-5515/47/i=6/a=S03.

[7] M.F.F. Nave, J. Rapp, T. Bolzonella, R. Dux, M.J. Mantsinen, R. Budny, P. Dumortier, M. von Hellermann, S. Jachmich, H.R. Koslowski, G. Maddison, A. Messiaen, P. Monier-Garbet, J. Ongena, M.E. Puiatti, J. Strachan, G. Telesca, B. Unterberg, M. Valisa, P. de Vries, and contributors to the JET-EFDA Workprogramme. Role of sawtooth in avoiding impurity accumulation and maintaining good confinement in JET radiative mantle discharges. Nuclear Fusion, 43(10):1204, 2003. http://stacks.iop.org/0029-5515/43/i=10/a=023.

[8] I T Chapman, S D Pinches, J P Graves, R J Akers, L C Appel, R V Budny, S Coda, N J Conway, M de Bock, L-G Eriksson, R J Hastie, T C Hender, G T A Huysmans, T Johnson, H R Koslowski, A Kramer-Flecken, M Lennholm, Y Liang, S Saarelma, S E Sharapov, I Voitsekhovitch, the MAST, TEXTOR Teams, and JET EFDA Contributors. The physics of sawtooth stabilization. Plasma Physics and Controlled Fusion, 49(12B):B385, 2007. http://stacks.iop.org/0741-3335/49/ $i=12 B / a=S 35$.

[9] I T Chapman. Controlling sawtooth oscillations in tokamak plasmas. Plasma Physics and Controlled Fusion, 53(1):013001, 2011. http://stacks .iop.org/0741-3335/53/i=1/a=013001.

[10] A Muck, T P Goodman, M Maraschek, G Pereverzev, F Ryter, H Zohm, and ASDEX Upgrade 
Team. Sawtooth control experiments on ASDEX Upgrade. Plasma Physics and Controlled Fusion, 47(10):1633, 2005. http://stacks.iop.org/0741-3335/47/i=10/a=004.

[11] L.-G. Eriksson, T. Johnson, M.-L. Mayoral, S. Coda, O. Sauter, R.J. Buttery, D. McDonald, T. Hellsten, M.J. Mantsinen, A. Mueck, J.-M. Noterdaeme, M. Santala, E. Westerhof, P. de Vries, and JET-EFDA contributors. On ion cyclotron current drive for sawtooth control. Nuclear Fusion, 46(10):S951, 2006. http://stacks .iop.org/0029-5515/46/i=10/a=S12.

[12] J. P. Graves, I. Chapman, S. Coda, L.-G. Eriksson, and T. Johnson. Sawtooth-control mechanism using toroidally propagating ion-cyclotron-resonance waves in tokamaks. Phys. Rev. Lett., 102(6):065005, Feb 2009. http://prl.aps.org/abstract/PRL/v102/i6/e065005.

[13] I.T. Chapman, V.G. Igochine, J.P. Graves, S.D. Pinches, A. Gude, I. Jenkins, M. Maraschek, G. Tardini, the ASDEX Upgrade Team, and JET EFDA Contributors. Sawtooth control and the interaction of energetic particles. Nuclear Fusion, 49(3):035006, 2009. http://stacks .iop. org $/ 0029-5515 / 49 / i=3 / a=035006$.

[14] J.P. Graves, I.T. Chapman, S. Coda, T. Johnson, M. Lennholm, B. Alper, M. de Baar, K. Crombe, L.-G. Eriksson, R. Felton, D. Howell, V. Kiptily, H.R. Koslowski, M.-L. Mayoral, I. Monakhov, I. Nunes, S.D. Pinches, and JET-EFDA Contributors. Experimental verification of sawtooth control by energetic particles in ion cyclotron resonance heated JET tokamak plasmas. Nuclear Fusion, 50(5):052002, 2010. http://stacks.iop.org/0029-5515/50/i=5/a=052002.

[15] V Igochine, I T Chapman, V Bobkov, S Günter, M Maraschek, D Moseev, G Pereversev, M Reich, J Stober, and ASDEX Upgrade team. Destabilization of fast particle stabilized sawteeth in asdex upgrade with electron cyclotron current drive. Plasma Physics and Controlled Fusion, 53(2):022002, 2011. http://stacks.iop.org/0741-3335/53/i=2/a=022002.

[16] J I Paley, F Felici, S Coda, T P Goodman, and the TCV Team. From profile to sawtooth control: developing feedback control using ecrh/eccd systems on the tcv tokamak. Plasma Physics and Controlled Fusion, 51(12):124041, 2009. http://stacks.iop.org/0741-3335/51/i=12/a= 124041.

[17] F Porcelli. Fast particle stabilisation. Plasma Physics and Controlled Fusion, 33(13):1601, 1991. http://stacks.iop.org/0741-3335/33/i=13/a=009.

[18] John Wesson. Tokamaks. Clarendon Press, Oxford, third edition, 2004. http://www.iop.org/EJ/ abstract/0741-3335/46/3/173515.

[19] B. B. Kadomtsev. On disruptive instability in tokamaks. Soviet Journal of Plasma Physics, 1:710715, oct 1975. http://adsabs.harvard.edu/abs/1975SvJPP...1Q.710K.

[20] V. Igochine, O. Dumbrajs, D. Constantinescu, H. Zohm, G. Zvejnieks, and the ASDEX Upgrade Team. Stochastization as a possible cause for fast reconnection during MHD mode activity in the ASDEX Upgrade tokamak. Nuclear Fusion, 46(7):741-751, 2006. http://www . iop.org/EJ/abstract/0029-5515/46/7/006.

[21] V. Igochine, O. Dumbrajs, H. Zohm, A. Flaws, and the ASDEX Upgrade Team. Stochastic sawtooth reconnection in ASDEX Upgrade. Nuclear Fusion, 47(1):23-32, 2007. http://iopscience.iop. org/0029-5515/47/1/004.

[22] O. Dumbrajs, V. Igochine, H. Zohm, and the ASDEX Upgrade Team. Diffusion in a stochastic magnetic field in ASDEX Upgrade. Nuclear Fusion, 48(2):024011 (7pp), 2008. http://www. iop. org/EJ/abstract/0029-5515/48/2/024011.

[23] Xiaoyuan Xu, Jun Wang, Yizhi Wen, Yi Yu, Adi Liu, Tao Lan, Changxuan Yu, Baonian Wan, Xiang Gao, Youwen Sun, N C Luhmann Jr, C W Domier, Z G Xia, and Zuowei Shen. Experimental investigation of $\mathrm{m} / \mathrm{n}=1 / 1$ and high-order harmonic modes during the sawtooth oscillation in a low beta tokamak plasma. Plasma Physics and Controlled Fusion, 52(1):015008, 2010. http://stacks.iop.org/0741-3335/52/i=1/a=015008.

[24] A.J. Lichtenberg. Stochasticity as the mechanism for the disruptive phase of the $\mathrm{m}=1$ tokamak oscillations. Nuclear Fusion, 24(10):1277, 1984. http://stacks.iop.org/0029-5515/24/i=10/ $\mathrm{a}=004$.

[25] A.J. Lichtenberg, K. Itoh, S.-I. Itoh, and A. Fukuyama. The role of stochasticity in sawtooth oscillations. Nuclear Fusion, 32(3):495, 1992. http://stacks.iop.org/0029-5515/32/i=3/a= 
I12.

[26] Kaiyun Chen, Liqun $\mathrm{Hu}$, Yanmin Duan, and Tianpeng Ma. Tomography analysis of the sawtooth crash with post-cursor in LHCD plasma on HT-7 tokamak. Physics Letters A, 372(24):4469 - 4473, 2008. http://www.sciencedirect.com/science/article/ B6TVM-4S92T J6-1/2/7f365494e84c15ae2b136fbb96226820.

[27] J. A. Wesson, B. Alper, A. W. Edwards, and R. D. Gill. Transport in the sawtooth collapse. Phys. Rev. Lett., 79(25):5018-5021, Dec 1997. http://prola.aps.org/abstract/PRL/v79/i25/ p5018_1.

[28] H. K. Park, E. Mazzucato, Jr. N. C. Luhmann, C. W. Domier, Z. Xia, T. Munsat, A. J. H. Donne, I. G. J. Classen, M. J. van de Pol, and TEXTOR team. Self-organized $T_{e}$ redistribution during driven reconnection processes in high-temperature plasmas. Physics of Plasmas, 13(5):055907, 2006. http://link.aip.org/link/?PHP/13/055907/1.

[29] V. Igochine, O. Dumbrajs, H. Zohm, and the ASDEX Upgrade Team. Transition from quasiperiodicity to chaos just before sawtooth crash in the ASDEX Upgrade tokamak. Nuclear Fusion, 48(6):062001 (5pp), 2008. http://stacks.iop.org/0029-5515/48/062001.

[30] V. Igochine, O. Dumbrajs, H. Zohm, G. Papp, G. Por, G. Pokol, and the ASDEX Upgrade Team. The role of stochastization in fast MHD phenomena on ASDEX Upgrade. In 23st IAEA Fusion Energy Conference, volume EX/P9-10, Genf, Switzerland, 2008. http://www-pub.iaea.org/ MTCD/Meetings/FEC2008/ex_p9-10.pdf.

[31] G. Papp, G. Pokol, G. Por, V. Igochine, and ASDEX Upgrade Team. Analysis of sawtooth precursor activity in ASDEX Upgrade using bandpower correlation method. In Europhysics Conference Abstracts, volume 33E, page P1.157, 2009. http://epsppd.epfl.ch:80/Sofia/pdf/P1_157.pdf.

[32] Youwen Sun, Baonian Wan, Liqun Hu, Kaiyun Chen, Biao Shen, and Jianshan Mao. Observation of heat transfer across $\mathrm{x}$ point of the islands during sawtooth crash on the HT-7 tokamak. Plasma Physics and Controlled Fusion, 51(6):065001 (11pp), 2009. http://stacks.iop.org/0741-3335/ 51/065001/.

[33] R.J. Buttery, T.C. Hender, D.F. Howell, R.J. La Haye, O. Sauter, D. Testa, and contributors to the EFDA-JET Workprogramme. Onset of neoclassical tearing modes on JET. Nuclear Fusion, 43(2):69, 2003. http://stacks.iop.org/0029-5515/43/i=2/a=301.

[34] H. G. Schuster and W. Just. Deterministic chaos. Wiley-VCH, Germany, 4th edition, 2004.

[35] V. Igochine, A. Gude, M. Maraschek, and ASDEX Upgrade Team. Hotlink based soft X-ray diagnostic on ASDEX Upgrade. Technical Report 1/338, Max Planck Insitut für Plasmaphysik, (IPP), Garching bei München, May 2010. http://edoc.mpg.de/display.epl?mode=doc\&id= 476537.

[36] Stephane Mallat. A wavelet tour of signal processing. Academic Press, second edition, 2001.

[37] P. Varela. Automatic time-frequency analysis for plasma density profile evaluation from microwave reflectometry. PhD thesis, Universidade Técnica de Lisboa, 2002.

[38] E. W. Dijkstra. A note on two problems in connexion with graphs. Numerische Mathematik, 1:269271, 1959. http://www-m3.ma.tum.de/foswiki/pub/MN0506/WebHome/dijkstra.pdf.

[39] G. Pokol, G. Por, S. Zoletnik, and W7-AS team. Application of a bandpower correlation method to the statistical analysis of MHD bursts in quiescent Wendelstein-7 AS stellarator plasmas. Plasma Physics and Controlled Fusion, 49(9):1391-1408, 2007. http://www.iop.org/EJ/abstract/ 0741-3335/49/9/003/.

[40] A. Gude and M. Maraschek. Private communication, 2010.08.

[41] H Soltwisch and H R Koslowski. Sawtooth modulation of the poloidal field in TEXTOR under ohmic heating conditions. Plasma Physics and Controlled Fusion, 37(6):667-678, 1995. http: //stacks. iop.org/0741-3335/37/667.

[42] M. Brix, N. C. Hawkes, A. Boboc, V. Drozdov, S. E. Sharapov, and JET-EFDA Contributors. Accuracy of EFIT equilibrium reconstruction with internal diagnostic information at JET. In PROCEEDINGS OF THE 17TH TOPICAL CONFERENCE ON HIGH-TEMPERATURE PLASMA DIAGNOSTICS (HTPD0), volume 79, page 10F325. AIP, 2008. http://link.aip. org/link/?RSI/79/10F325/1. 
[43] C G Gimblett and R J Hastie. Calculation of the post-crash state and $11 / 2 \mathrm{~d}$ simulation of sawtooth cycles. Plasma Physics and Controlled Fusion, 36(9):1439, 1994. http://stacks.iop. org $/ 0741-3335 / 36 / i=9 / a=005$.

[44] Young C. Kim and Edward J. Powers. Digital bispectral analysis and its applications to nonlinear wave interactions. Plasma Science, IEEE Transactions on, 7(2):120-131, June 1979. http: //dx.doi.org/10.1109/TPS.1979.4317207.

[45] G. Pokol, G. Papp, G. Por, S. Zoletnik, A. Weller, and W7-AS team. Experimental study and simulation of W7-AS transient MHD modes. AIP Conference Proceedings, 993(1):215-218, 2008. http://link. aip.org/link/?APC/993/215/1.

[46] G.I. Pokol, N. Lazanyi, G. Por, A. Magyarkuti, G. Papp, A. Gude, V. Igochine, M. Maraschek, and ASDEX-Upgrade Team. A wavelet based method for detecting transient plasma waves and determining their spatial structure. In Europhysics Conference Abstracts, volume 34A, page P5.129, Dublin, Ireland, 2010. http://ocs.ciemat.es/EPS2010PAP/pdf/P5.129.pdf.

[47] M. Schittenhelm and H. Zohm. Analysis of coupled MHD modes with Mirnov probes in ASDEX Upgrade. Nuclear Fusion, 37(9):1255, 1997. http://stacks.iop.org/0029-5515/37/i=9/a= I06.

[48] I. T. Chapman, R. Scannell, W. A. Cooper, J. P. Graves, R. J. Hastie, G. Naylor, and A. Zocco. Magnetic reconnection triggering magnetohydrodynamic instabilities during a sawtooth crash in a tokamak plasma. Phys. Rev. Lett., 105(25):255002, Dec 2010. http://prl.aps.org/abstract/ PRL/v105/i25/e255002.

[49] V. Igochine, J. Boom, I. Classen, O. Dumbrajs, S. Gunter, K. Lackner, G. Pereverzev, H. Zohm, and ASDEX Upgrade Team. Structure and dynamics of sawteeth crashes in asdex upgrade. Physics of Plasmas, 17(12):122506, 2010. http://link.aip.org/link/?PHP/17/122506/1.

[50] R. J. Hastie, T. C. Hender, B. A. Carreras, L. A. Charlton, and J. A. Holmes. Stability of ideal and resistive internal kink modes in toroidal geometry. Physics of Fluids, 30(6):1756-1766, 1987. http://link. aip.org/link/?PFL/30/1756/1.

[51] Andreas Letsch, Hartmut Zohm, Francois Ryter, Wolfgang Suttrop, Anja Gude, Franco Porcelli, Clemente Angioni, and Ivo Furno. Incomplete reconnection in sawtooth crashes in ASDEX Upgrade. Nuclear Fusion, 42(9):1055, 2002. http://stacks.iop.org/0029-5515/42/i=9/a= 302.

[52] RJ Hastie. Sawtooth instability in tokamak plasmas. Astrophysics and space science, 256(1-2):177204, 1998. 